\title{
PCaGuard: A Software Platform to Support Optimal Management of Prostate Cancer
}

\author{
Ioannis Tamposis ${ }^{1}$ Ioannis Tsougos ${ }^{2}$ Anastasios Karatzas ${ }^{3}$ Katerina Vassiou ${ }^{4}$ Marianna Vlychou ${ }^{5}$ \\ Vasileios Tzortzis ${ }^{3}$
}

${ }^{1}$ Department of Computer Science and Biomedical Informatics, University of Thessaly, Lamia, Greece

2 Department of Medical Physics, Medical School, University of Thessaly, Larisa, Greece

${ }^{3}$ Department of Urology, Medical School, University of Thessaly, Larisa, Greece

${ }^{4}$ Radiology and Anatomy Department, Medical School, University of Thessaly, Larisa, Greece

${ }^{5}$ Radiology Department, Medical School, University of Thessaly, Larisa, Greece

Appl Clin Inform 2022;13:91-99.

\begin{abstract}
Address for correspondence Ioannis Tamposis, Department of Computer Science and Biomedical Informatics, University of Thessaly, Papasiopoulou 2-4, Lamia 35100, Greece (e-mail: itamposis@uth.gr).
\end{abstract}

\section{Abstract}

Keywords

- prevention

- diagnosis

- clinical workflow

- prostate cancer

- multiparametric MRI-U/S fusion

- health care system framework

received

July 28, 2021

accepted after revision

November 24, 2021
Background and Objective Prostate cancer (PCa) is a severe public health issue and the most common cancer worldwide in men. Early diagnosis can lead to early treatment and long-term survival. The addition of the multiparametric magnetic resonance imaging in combination with ultrasound (mpMRI-U/S fusion) biopsy to the existing diagnostic tools improved prostate cancer detection. Use of both tools gradually increases in every day urological practice. Furthermore, advances in the area of information technology and artificial intelligence have led to the development of software platforms able to support clinical diagnosis and decision-making using patient data from personalized medicine.

Methods We investigated the current aspects of implementation, architecture, and design of a health care information system able to handle and store a large number of clinical examination data along with medical images, and produce a risk calculator in a seamless and secure manner complying with data security/accuracy and personal data protection directives and standards simultaneously. Furthermore, we took into account interoperability support and connectivity to legacy and other information management systems. The platform was implemented using open source, modern frameworks, and development tools. Results The application showed that software platforms supporting patient follow-up monitoring can be effective, productive, and of extreme value, while at the same time, aiding toward the betterment medicine clinical workflows. Furthermore, it removes access barriers and restrictions to specialized care, especially for rural areas, providing the exchange of medical images and patient data, among hospitals and physicians. Conclusion This platform handles data to estimate the risk of prostate cancer detection using current state-of-the-art in eHealth systems and services while fusing emerging multidisciplinary and intersectoral approaches. This work offers the research community an open architecture framework that encourages the broader adoption of more robust and comprehensive systems in standard clinical practice.

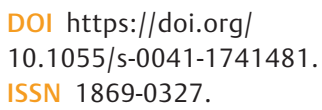

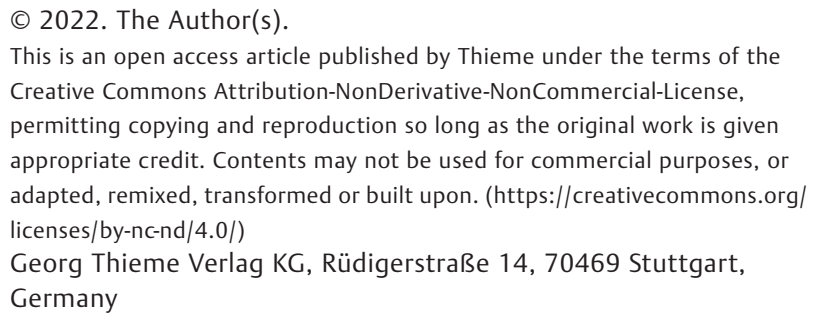




\section{Background and Significance}

Prostate cancer (PCa) is a serious public health problem being the most common type of cancer and the second most common cause of cancer death among men in most developed countries. ${ }^{1}$ Increased incidence and mortality negatively affect health care systems on both economic and social levels. Despite the advances of cancer biology, diagnosis of prostate cancer remains problematic. Early diagnosis of prostate carcinoma when it is confined to the prostate is vital to provide effective treatment and thereby reducing mortality. ${ }^{2}$ To date, prostate cancer is diagnosed by digital rectal examination (DRE; cancer detection is less than $2 \%)^{3}$ and prostate-specific antigen (PSA). PSA is the best tumor marker used for early detection of PCa, although it has moderate sensitivity (20\% of patients with prostate cancer have normal PSA) and specificity (two in three with increased PSA do not have biopsy cancer). To increase PSA testing specificity, other metrics have been sought such as free-to-total PSA and prostate health index (PHI). ${ }^{4}$ Guidelines suggest transrectal ultrasound-guided biopsy (TRUS-biopsy) in men who present with an increased serum PSA or a suspicious DRE. Standard TRUS biopsy protocols consist of systematic 10- to 12-needle core biopsies from the entire gland. ${ }^{5}$ This may result to overdiagnosis and consequently overtreatment of clinically insignificant cancers. On the other hand, clinically significant cancers are occasionally missed. In addition, many men without cancer might be exposed to unnecessary biopsies, with the risk of severe complications. $^{6}$

Recently, multiparametric magnetic resonance imaging, in combination with ultrasound evaluation and biopsy (mpMRI-U/S fusion) improved detection of prostate cancer and are increasingly used in urological practice. ${ }^{7}$ Furthermore, mpMRI is used as a diagnostic test to decide which men with an elevated PSA go on to biopsy leading to reduce unnecessary TRUS biopsies and improve diagnostic accuracy. ${ }^{8}$ Thus, mpMRI-U/S fusion tends to modify the prostate cancer clinical practice, as it detects higher risk carcinomas and overlooks low-risk entities,,${ }^{9,10}$ making it as a potential diagnostic test ${ }^{11,12}$ and modifying the prostate cancer clinical pathway. ${ }^{13-18}$

Advances in the areas of information technology and artificial intelligence have leaded to the development of software platforms that support clinical diagnosis and decision-making using patient data from personalized medicine. Clinical decision support systems (CDSSs) aim to codify and strategically manage biomedical knowledge to handle challenges in clinical practice using mathematical modeling tools, medical data processing techniques, or artificial intelligence methods. CDSSs cover a wide range of applications, from diagnosis support to modeling, the probability of occurrence of various diseases, or the efficiency of alternative therapeutic schemes. ${ }^{19,20}$ To do so, CDSSs are using not only individual patient data, but also data on risk factors and efficiency of available therapeutic schemes stored in databases. This way, CDSSs provide clinicians with patient-specific assessments or recommendations to aid clinical decision-making. The majority of published studies regard- ing CDSS systems for PCa support, concern computer-aided diagnosis systems based on PSA, ${ }^{21}$ or patient characteristics (e.g., weight, height, age, and if smoking). ${ }^{22,23}$ To the best of our knowledge, however, a similar CDSS based on mpMRI-U/S fusion technology for supporting and improving PCa screening and follow-up like the proposed PCaGuard system has not been reported in the literature.

Because health care is a complex ecosystem of interconnected stakeholders, medical devices, and various subsystems to deliver end-to-end patient care, the need for more efficient and sophisticated systems which include workflow aspects is essential ${ }^{24,25}$ to achieve efficient and continuous patient monitoring. The design and implementation of CDSS present significant challenges, such as involving specialists from various health disciplines, that is, urologists performing PSA tests, biologists conducting molecular tests, along with the other data and pathologists diagnosing biopsies' material. ${ }^{24}$ Additionally, the security requirements are extremely strict because of the handling of sensitive personal data and medical records, and, finally, user training and acceptance, especially when such systems are deployed for the first time, are very crucial.

We present the design and implementation of PCaGuard, a software platform based on latest technologies for supporting PCa detection using and processing clinical (DRE), biochemical (PSA and derivates), imaging (transrectal ultrasound and mpMRI) and histopathologic findings from mpMRI-U/S fusion biopsy. In addition, we advance the current state-of-the-art in eHealth systems and services fusing emerging multidisciplinary and intersectoral approaches to deliver an open architecture framework that will expedite the wider adoption of such systems in standard clinical practice.

\section{Methods}

We investigated present aspects of the interoperability, accessibility, architecture, and design of a health care information system able to handle and store a large number of clinical examination data along with medical images. The system stores and processes findings from clinical (DRE), biochemical (PSA and derivates), imaging (transrectal ultrasound and mpMRI) and histopathological methods (mpMRI-U/S fusion biopsy), along with patient medical history and nonmedical data such as demographics. Emphasis will be given to the critical aspects of the informational infrastructure such as data anonymization tools, patient identification components, data integration, and communication toolkits, as well as additional tools for efficient management of high-volume computational data. Additional software tools will be designed and developed to ensure data security and data integrity.

\section{Interoperability in Health}

Interoperability has been an important issue in health care for over a decade and is one of the key aspects of health care systems. ${ }^{26}$ A system should be compliant with international open standards for exchange and medical data access. ${ }^{27}$ 
Appropriate methods are proposed by the agency for integration, diffusion, and archiving of medical information (AIDA). ${ }^{28} \mathrm{~A}$ lack of interoperability prohibits communication which can lead to missed opportunities to inform better clinical and administrative decisions. The Health Level Seven (HL7) and Digital Imaging and Communications in Medicine (DICOM) are the two most common standards used in the communication of medical data and medical imaging. The HL7 ()$^{29}$ is a standard for medical data exchange among different healthcare information systems. DICOM ${ }^{30}$ is the standard for the encoding, the management and the exchange of medical imaging. DICOM is implemented in almost every radiology device to produce, manage, and distribute their medical images. The majority of existing standards regarding data and communications for health care support enables better communication and allows data transfer among different systems accurately, effectively, and on time. On the other hand, it is unclear to the practical implementer of how the standard can best be applied to a specific situation. In 1997, the Integrating the Healthcare Enterprise (IHE) was introduced to provide imaging workflows and help a hospital IT infrastructure to improve the integration of imaging data. $\mathrm{IHE}^{31}$ is an organization consisting of health professionals and industry organizations; aims to improve IT systems to exchange health care information. IHE recommends the coordinated use of health standards such as DICOM and HL7 to support best patient care.

\section{Privacy Protection}

An important aspect is that all the above services have to be provided and applied in accordance with the law. Nowadays, two laws are related to the health issue, the first is the Health Insurance Portability and Accountability Act (HIPAA), a U.S. based law of 1996, and the second is the General Data Protection Regulation (GDPR) 2016/679 adopted by the European Union. Further, by European Directive, member states shall protect the fundamental rights and freedoms of citizens, and, in particular, their right to privacy, with respect to the processing of personal data.

\section{Software Description}

This section presents the basic software technologies and frameworks used for the implementation of the platform. The synthesis of the platform software is based on various components. Combined they create a framework capable to host medical applications designed for specific health sectors. Key components include web interfaces, specific frameworks capable to retrieving/communicating data from/to subsystems, and the database used to store data and specialized toolkits to store and exchange medical images. All described components use open source or no-cost license (making available for researchers), comply with international certificates and base on a leading technology.

\section{Framework}

The basic components include virtual machine, database, application server, and picture archiving and communication system (PACS). The framework representation of this hybrid system is shown in -Fig. 1. The design of the integrated platform uses virtualization technologies using VMware ESXi $^{32}$ to enable the health sector simplify the necessary infrastructure and the whole system be cloud ready. Specifically, two basic components used are the Apache Tomcat as application server and Oracle database Express Edition (XE) to store all medical data. The application is web based and was implemented with the oracle application express, an application development platform to build secure and complex web and mobile applications using popular JavaScript frameworks and the reports in PDF format were generated with the BIRT library. ${ }^{12}$ The dcm 4 che toolkit ${ }^{33}$ is used for archiving DICOM images and communicating with medical modalities. Dcm4che is a well-known open-source implementation of the DICOM standard that collaborates completely with diagnostic viewers like OsiriX. ${ }^{34}$ Furthermore, PCaGuard provides basic DICOM services and interacts with other systems through the HL7 protocol and web services.

The complexity and large variety of data from different domains (e.g., cardiology and urology) in the case of examinations and their respective results (different number of fields, different meaning, and different information) led us to use entity-attribute-value (EAV) approach for modeling highly heterogeneous data, for example, patient visits, examination results, laboratory reports, therapies, and medications. Due to the EAV design of the database, the workflow for adding a new examination into the system is straightforward and guides the user. With this approach, each new examination (with different structure) can be rapidly developed keeping the barrier low for developing new functions.

Finally, additional system functionalities include transmission of informative SMS texts to patient cell phones; this is performed only after the urologist managing the patient requests to release such messages. This functionality is important to urologists, the other option would be a telephone call to the patient, to inform them about the availability of results or remind scheduled appointment. An additional option may involve the transmission of the examination results to the patient, in case that they are negative, and thus no additional visit to the hospital is required.

\section{Security}

The entire platform provides security using an integrated firewall and web application using SSL encryption. All users are enforced to use strong passwords following complexity policy requirements in usernames and passwords and to change their passwords on initial login and every determined time thereafter. The system is configured to automatically logged out users, after 90 minutes of inactivity. A rolebased access control (RBAC) mechanism is used to protect sensitive data and ensure system access to authorized users. Furthermore, anonymization process removes sensitive personal information from the data and medical images, for users with limited rights either to view clinical data or to export data for research purposes. Finally, the system keeps audit logs, to performs control into which data has been accessed and shared by each user. 


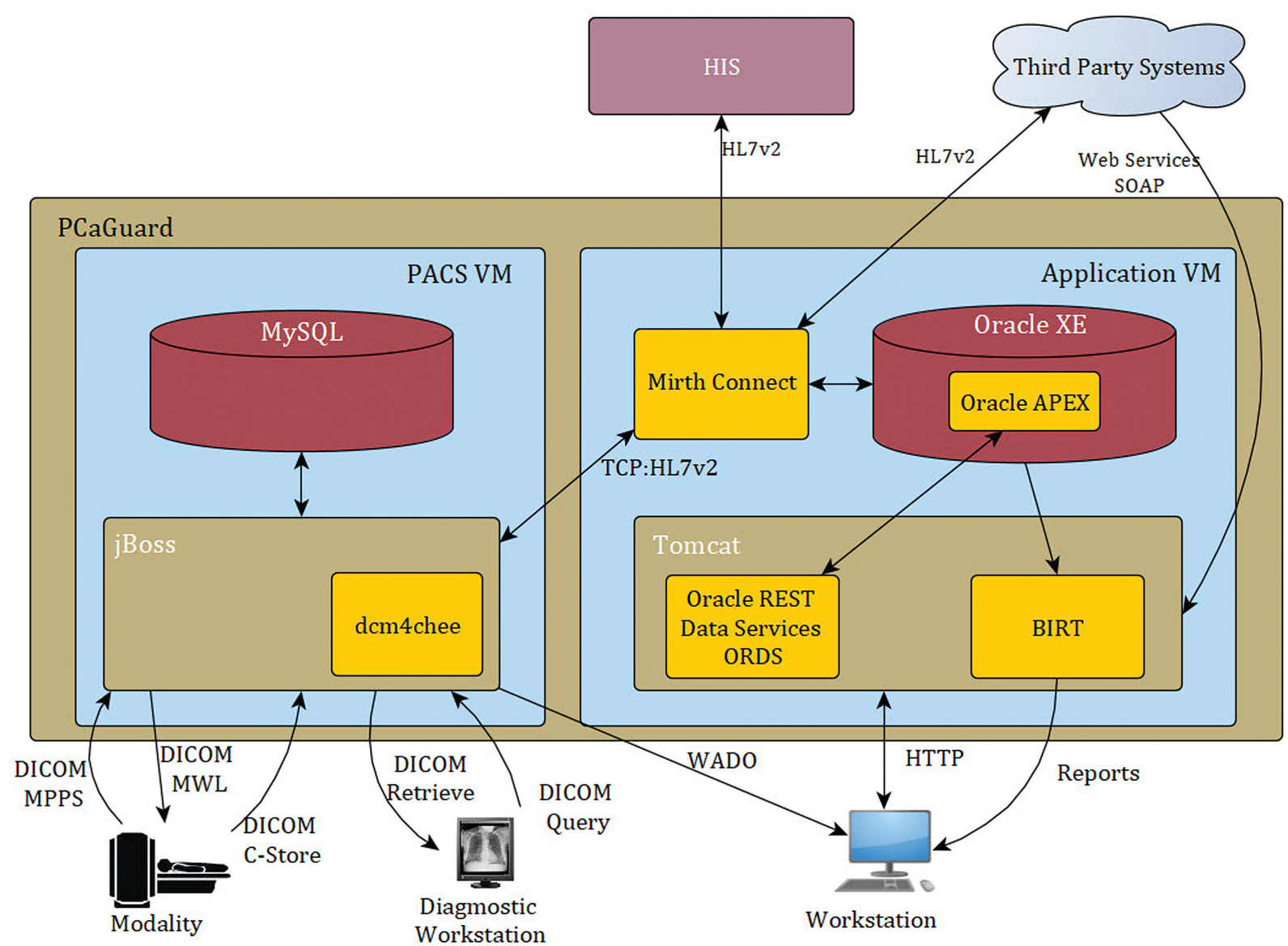

Fig. 1 Diagram of PCaGuard physical architecture. The synthesis of the platform software is based on various components. The framework includes PACS component to store and exchange medical images, Mirth component to retrieving/communicating data from/to subsystems, Oracle database Express Edition to store data, Apache Tomcat as application server, BIRT component to generate reports. Furthermore, PCaGuard provides basic DICOM services and interacts with other systems through the HL7 protocol and web services. APEX, Application Express; BIRT, Business Intelligence Reporting Tool; DICOM, Digital Imaging and Communications in Medicine; HIS, hospital information system; HL7, Health Level Seven; HTTP, HyperText Transfer Protocol; MPPS, modality performed procedure step; MWL, modality work list; ORDS, Oracle REST Data Services; PACS, picture archiving and communication system; REST, representational state transfer; SOAP, simple object access protocol; VM, Virtual Machine; WADO, web access to DICOM object; XE, express edition.

\section{Interoperability}

Mirth connect component is used to communicate with other subsystems of the hospital IT system (hospital information system [HIS] and laboratory information system [LIS]) using HL7 protocol to handle examination orders and to retrieve and request clinical information. ${ }^{35}$ Additionally, the system can be securely interconnected with a governmental organization (the Greek national health service) using a simple object access protocol (SOAP) web service integration component to receive accurate patient data through the social security number (SSN) and to the national prescription system to allow physicians to perform prescriptions of examinations in an organized, controlled, and recorded manner. Furthermore, system implements web access to DICOM object (WADO) functionality, according to the standard specifications, to allow querying medical images by patient identifier or other information.

The system to store and manage medical images interacts with available medical modalities that support DICOM integration standard. ${ }^{36}$ System workflow processes and transactions are in conformance with the IHE profiles (e.g., Scheduled Workflow, Patient Information Reconciliation, Post-Processing Workflow). ${ }^{5}$ According to IHE, the system provides the DICOM modality work list (MWL), the modality performed procedure step (MPPS) DICOM message, and DICOM C-Store to send the medical images. Additionally, the system is designed to provide remote capabilities of telediagnosis to removes barriers and restrictions in access to specialized care, especially for rural areas units that lack the necessary medical equipment and specialized medical staff. In fact, in that case, the modality can be locally operated by technologists while diagnosis can be performed remotely by radiologists. Finally, the framework is DICOM and IHE compliant.

\section{Picture Archiving and Communication System Functionality}

PCaGuard integrates a PACS component which is vendor neutral to handle and store medical images using DICOM and HL7 standards. Exist component provides functions for querying, acquiring, deploying, and retrieving medical images. PCaGuard PACS component uses the robust and open-source 
$\mathrm{dcm} 4 \mathrm{che}$ toolkit ${ }^{33}$ to access DICOM functionality and cooperate completely with OsiriX diagnostic viewer. ${ }^{34}$

\section{Image Viewing Functionality}

PCaGuard incorporates and collaborates with various kinds of viewers to provide radiologists and clinicians all necessary tools for viewing imaging exams from any place with Internet access. First, for a diagnostic viewer, PCaGuard is OsiriX, ${ }^{34}$ ready as it collaborates with it, and provides full functionality. System also incorporates a web-based DICOM viewer that allows users to display medical images easily and quickly, providing basic functions such as zoom, contrast, rotate, and measurement tools. ${ }^{37}$

\section{Use Case}

The presented platform has been implemented in a production environment at the University Hospital of Larissa,
UHL, involving over 30 users (doctors, technologists, researchers, and secretaries). The selected patients who were recruited from a large screening group for prostate cancer underwent a multiparametric MRI scan with the same imaging protocol by the use of a 3-Tesla MR scanner (HDx, GE Healthcare). Prostate imaging and interpretation was based on prostate imaging reporting and data system version 2 (PI-RAD v2.1) as indicated by the recent international guidelines for prostate cancer. Suspicious cases underwent fusion-guided US/MRI transrectal biopsy. A total of 150 fusion-guided TRUS, mpMRI biopsies were performed. Of these, 45 were diagnosed as cancers of which 33 were clinically significant.

Further, a usage scenario is presented following the workflow presented in - Fig. 2. Men presented with an elevated serum PSA and/or suspicious digital rectal examinations are candidates for further evaluation. Eligible men were required to give written informed consent. Initially, only the SSN is

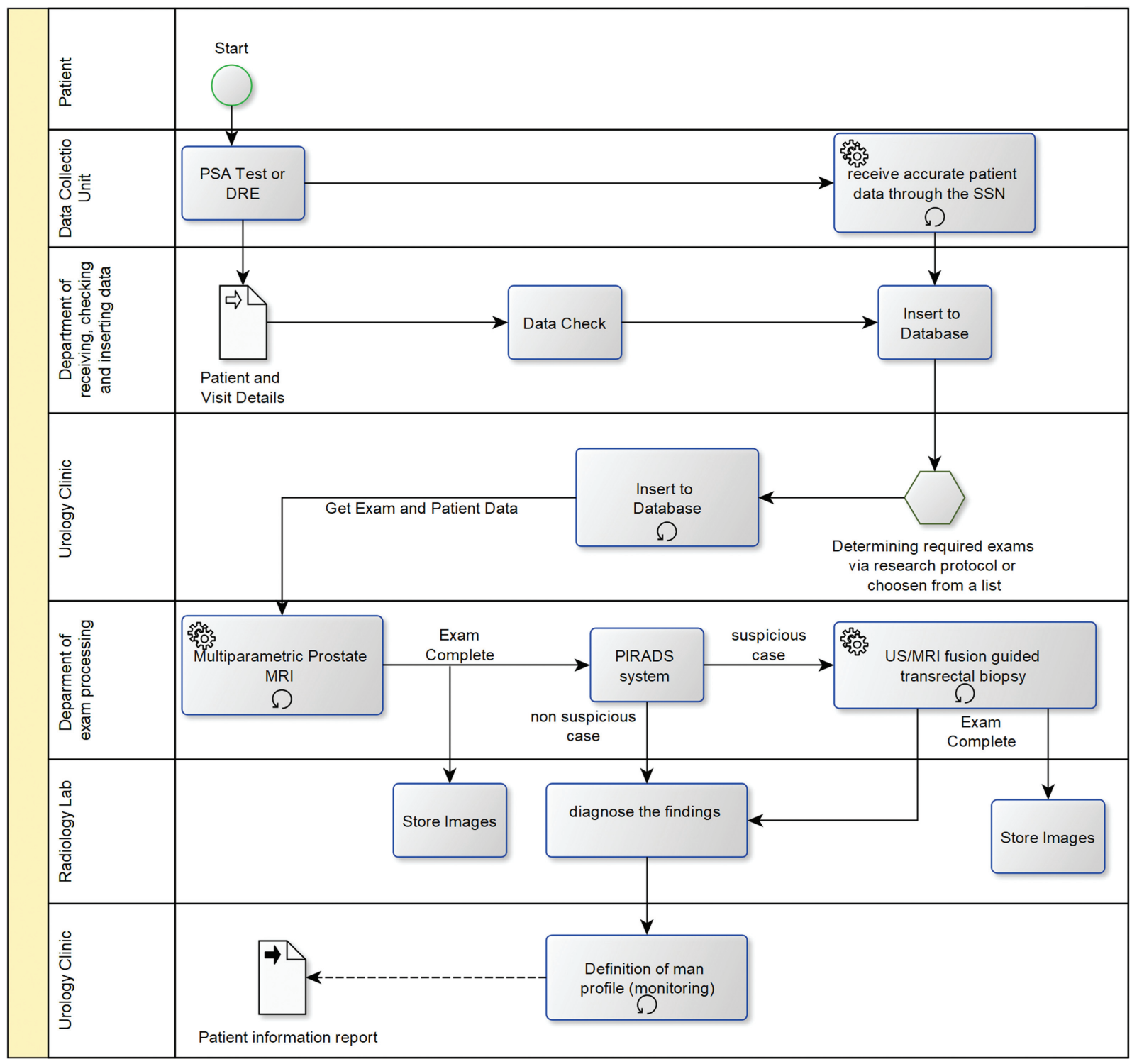

Fig. 2 Workflow activity diagram. DRE, digital rectal examination; PSA, prostate-specific antigen; MRI, magnetic resonance imaging; SSN, social security number; US, ultrasound. 
inserted in the system by the physician. All patient data are filled automatically through a national web service or HIS that provides all patient data via SSN. After registration, a new patient visit can be created by the physician, along with form filling with demographic and medical data, including lifestyle habits, medical history, and nutrition habits. The completion of a new visit ends with the request for examinations to be performed for this man. The list of examinations to be scheduled becomes automatically available to the radiologists at their workstations (mp-MRI scans). Patient order is automatically presented to the modality operator without the need of human interference. Upon multiparametric MRI scan completion, a report based on PIRADS (Prostate Imaging-Reporting and Data System) system V2.1 is provided. Only suspicious cases undergo a fusion U/S-MRIguided transrectal biopsy that is performed by urologists and radiologists at the Department of Radiology. On subsequent completion, the modality sends the images to the image archiving system (PACS) and automatically added in the patient record.

After the examination, a patient's study report is generated either by direct typing into a rich text editor or by dictating into a digital dictation system as presented in -Fig. 3. The final histological core-biopsy reports for each patient refer separately for the targeted area as indicated by the mpMRI and the right and the left prostate lobe. When the procedure is completed, it will be included in the patient record with its results (a medical report accompanied with medical data). In addition to that, any incident, including possible side effects during any of the above procedures will be included in patient's folder. Finally, the system has special functions for notifying doctors and other users or the patients by e-mail or SMS (e.g., for examination results).

\section{Discussion}

Several studies have already been presented in oncological cancer diseases to support clinical diagnosis and decisionmaking using patient data from personalized medicine. In the era of colorectal cancer, Becker et al have evaluated an open-source workflow system based on business process model and notation (BPMN) with unified medical language system (UMLS) for colorectal cancer screening. ${ }^{38}$ Since electronic health records (EHRs) contain more and more data, researchers develop methods to extract characteristics either from data ${ }^{39,40}$ or scanned and other outside documents contained in EHRs. ${ }^{41}$ In the market, data management systems available clinical, such as REDCap, OpenClinica, and eClinicalOS, but these systems are either commercial or not suitable for this project because the whole workflow is time consuming, error prone, and requires the integration of different components.

The presented architecture developed to produce an integrated software platform that combines all necessary functions and components to support the screening workflow and to collect, manage, and analyze clinical and administrative data in a seamless and secure manner complying at the same time with data security/accuracy and personal data protection directives and standards. Furthermore, the information technology (IT) platform provides a communication toolkit for interoperability support and connectivity to legacy and other information management systems including laboratory and clinical systems. Similar works have been implemented in the past such as PASS-PC, ${ }^{42}$ a web-based system aimed to collect a variety of data to support a research study for prostate cancer; PCCR, ${ }^{43}$ a multi-institutional webbased system aimed to collect a variety of data on pancreatic cancer patients; BCCR, ${ }^{44}$ a multicenter web-based system aimed to collect and manage a variety of data on breast cancer patients; and HPVGuard, ${ }^{19}$ a software platform to support management and prognosis of cervical cancer. All these solutions were developed using the integration of different components to support research programs in oncological cancer diseases.

\section{Conclusion}

The proposed architecture is implemented to support a research program able to evaluate patient prostate cancer development true risk through mpMRI-U/S fusion biopsy. The integrated PCaGuard platform offers several benefits to its users, including the following: (1) existing equipment integration, (2) use of standards for medical data and image handling, (3) data collection forms, (4) efficient workflows, (5) highest level network and data security, (6) various reports and paperless operation in all supported tasks, and (7) remote access. Platform use helped physicians acquire better clinical information toward more accurate and efficient diagnosis production.

The design and implementation of presented architecture was done using freely available software components. This is extremely important in health care systems and research community. These modern technologies can make applications available to users from a single point located in the Cloud thus facilitate maintenance or availability. The main focus of our future work is on developing services in the fields of clinical prediction, remote diagnosis and semantic data analysis, including integration with modern classification and prediction models (C/P models) or statistical models for classification and prediction processing, use of semantic web technologies for radiology image annotation, and report.

\section{Clinical Relevance Statement}

According to the literature searched, a CDSS based on mpMRI-U/S fusion technology for supporting and improving PCa screening and follow-up has not been reported. The complexity of a clinical workflow in a health care system it challenging to standardize efficient and sophisticated systems that are useful and achieve efficient and continuous monitoring of patients. Integrating open-source tools and no-cost license frameworks is a potential solution to some of the challenges in health care systems. Combining aspects of existing tools and available frameworks complying at the 

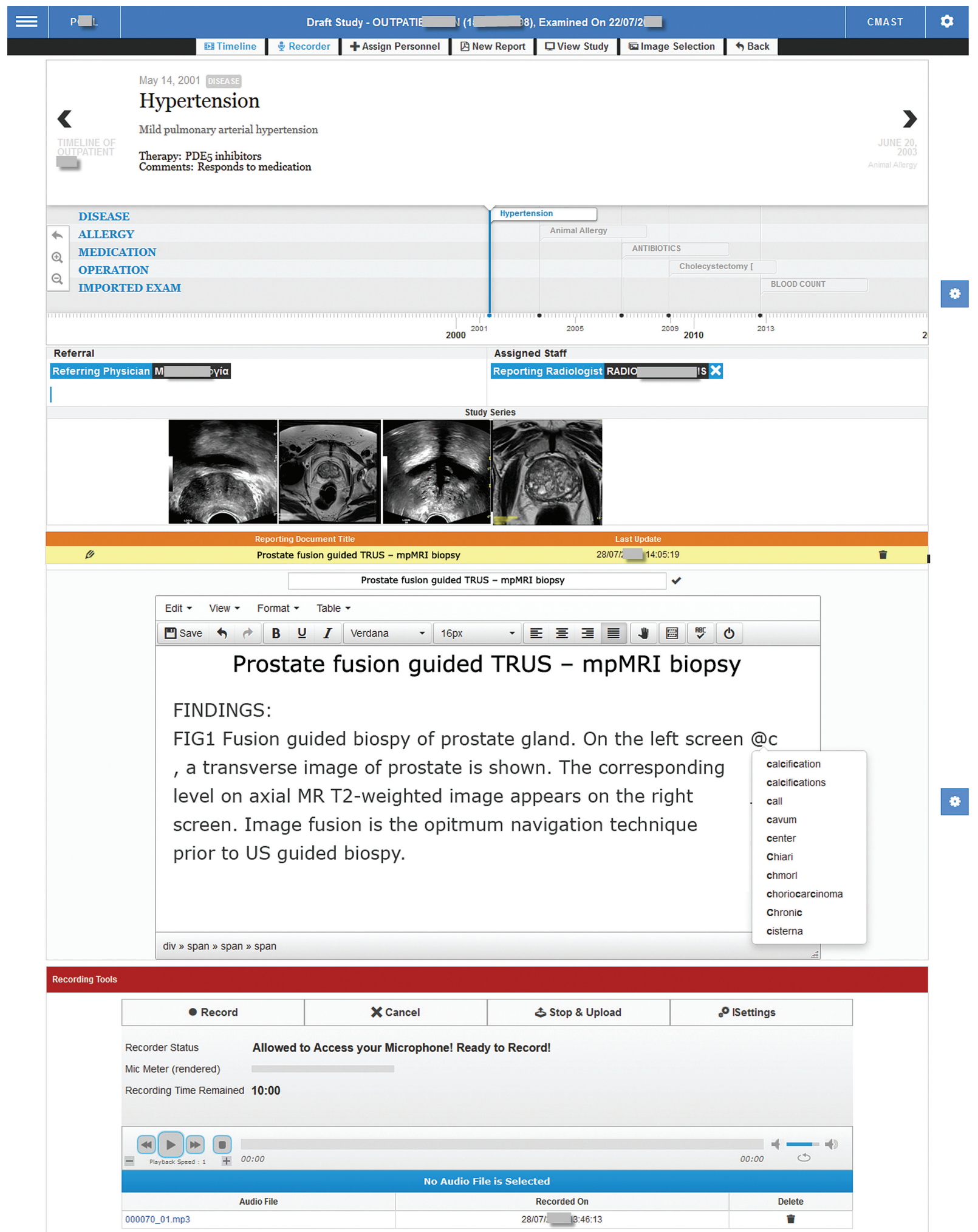

Fig. 3 PCaGuard's reporting application screen. mpMRI, multiparametric magnetic resonance imaging; TRUS, transrectal ultrasound-guided biopsy.

same time with data security/accuracy and personal data protection directives and standards provides the ability to develop a system for support clinical workflows and helps the application of standard clinical practice.

\section{Multiple Choice Questions}

1. Which of the following address specific clinical needs and workflows and eventually support best patient care? 
a. HL7 (Health Level Seven)

b. DICOM (Digital Imaging and Communications in Medicine)

c. IHE (Integrating the Healthcare Enterprise)

d. FHIR (Fast Healthcare Interoperability Resources)

Correct Answer: The correct answer is option c. IHE provides a common framework for building effective workflows and recommends the coordinated use of health standards such as DICOM and HL7.

2. Which system is suitable for storing and exchanging medical images?
a. LIS (laboratory information system)
b. PACS (picture archiving and communication system)
c. HIS (hospital information system)
d. CDSS (clinical decision support system)

Correct Answer: The correct answer is option b. A picture archiving and communication system (PACS) is a system used in medical imaging to store, retrieve, and distribute medical images using DICOM standard.

Protection of Human and Animal Subjects

The study was performed in compliance with the World Medical Association Declaration of Helsinki Ethical Principles for Medical Research Involving Human Subjects and was reviewed by the University Hospital Ethics Committee, Larisa Greece (reference 4861).

\section{Funding}

All authors report support from the European Union and Greek national funds through the Operational Program Competitiveness, Entrepreneurship and Innovation, under the call RESEARCH-CREATE-INNOVATE (project code: T1EDK-03264) for nonprofit. All authors report receipt from related university hospital of Larisa for nonprofit.

\section{Conflict of Interest}

None declared.

\section{References}

1 Sehn JK. Prostate cancer pathology: recent updates and controversies. Mo Med 2018;115(02):151-155

2 Litwin MS, Tan H-J. The diagnosis and treatment of prostate cancer: a review. JAMA 2017;317(24):2532-2542

3 Okotie OT, Roehl KA, Han M, Loeb S, Gashti SN, Catalona WJ. Characteristics of prostate cancer detected by digital rectal examination only. Urology 2007;70(06):1117-1120

4 Catalona WJ. Prostate cancer screening. Med Clin North Am 2018; 102(02):199-214

5 Harvey C, Pilcher J, Richenberg J, Patel U, Frauscher F. Applications of transrectal ultrasound in prostate cancer. Br J Radiol 2012;85 (special issue 1):S3-S17

6 Abraham NE, Mendhiratta N, Taneja SS. Patterns of repeat prostate biopsy in contemporary clinical practice. J Urol 2015;193 (04):1178-1184

7 Hegde JV, Mulkern RV, Panych LP, et al. Multiparametric MRI of prostate cancer: an update on state-of-the-art techniques and their performance in detecting and localizing prostate cancer. J Magn Reson Imaging 2013;37(05):1035-1054

8 Ahmed HU, El-Shater Bosaily A, Brown LC, et al; PROMIS study group. Diagnostic accuracy of multi-parametric MRI and TRUS biopsy in prostate cancer (PROMIS): a paired validating confirmatory study. Lancet 2017;389(10071):815-822

9 Turkbey B, Brown AM, Sankineni S, Wood BJ, Pinto PA, Choyke PL. Multiparametric prostate magnetic resonance imaging in the evaluation of prostate cancer. CA Cancer J Clin 2016;66(04): 326-336

10 Mowatt G, Scotland G, Boachie C, et al. The diagnostic accuracy and cost-effectiveness of magnetic resonance spectroscopy and enhanced magnetic resonance imaging techniques in aiding the localisation of prostate abnormalities for biopsy: a systematic review and economic evaluation. Health Technol Assess 2013;17 (20):vii-xix, 1-281

11 Valerio M, Willis S, van der Meulen J, Emberton M, Ahmed HU. Methodological considerations in assessing the utility of imaging in early prostate cancer. Curr Opin Urol 2015;25(06):536-542

12 Bossuyt PM, Irwig L, Craig J, Glasziou P. Comparative accuracy: assessing new tests against existing diagnostic pathways. BMJ 2006;332(7549):1089-1092

13 Fütterer JJ, Briganti A, De Visschere P, et al. Can clinically significant prostate cancer be detected with multiparametric magnetic resonance imaging? A systematic review of the literature. Eur Urol 2015;68(06):1045-1053

14 Siddiqui MM, Rais-Bahrami S, Turkbey B, et al. Comparison of MR/ultrasound fusion-guided biopsy with ultrasound-guided biopsy for the diagnosis of prostate cancer. JAMA 2015;313 (04):390-397

15 Schoots IG, Petrides N, Giganti F, et al. Magnetic resonance imaging in active surveillance of prostate cancer: a systematic review. Eur Urol 2015;67(04):627-636

16 Dickinson L, Ahmed HU, Hindley RG, et al. Prostate-specific antigen vs. magnetic resonance imaging parameters for assessing oncological outcomes after high intensity-focused ultrasound focal therapy for localized prostate cancer. Urol Oncol 2017;35 (01):30.e9-30.e15

17 Hegde JV, Demanes DJ, Veruttipong D, et al. Pretreatment 3T multiparametric MRI staging predicts for biochemical failure in high-risk prostate cancer treated with combination high-doserate brachytherapy and external beam radiotherapy. Brachytherapy 2017;16(06):1106-1112

18 Marks L, Young S, Natarajan S. MRI-ultrasound fusion for guidance of targeted prostate biopsy. Curr Opin Urol 2013;23(01):43-50

19 Tamposis I, Iordanidis E, Tzortzis L, et al. HPVGuard: A software platform to support management and prognosis of cervical cancer. IEEE2014:401-405; Athens, Greece

20 Klarenbeek SE, Weekenstroo HHA, Sedelaar JPM, Fütterer JJ, Prokop M, Tummers M. The effect of higher level computerized clinical decision support systems on oncology care: a systematic review. Cancers (Basel) 2020;12(04):1032

21 Mukai TO, Bro F, Olesen F, Vedsted P. To test or not: a registry-based observational study of an online decision support for prostatespecific antigen tests. Int J Med Inform 2013;82(10):973-979

$22 \mathrm{Yu} \mathrm{SH}$, Kim MS, Chung HS, et al. Early experience with Watson for Oncology: a clinical decision-support system for prostate cancer treatment recommendations. World J Urol 2021;39(02):407-413

23 Lin $\mathrm{H}-\mathrm{C}$, Wu H-C, Chang C-H, Li T-C, Liang W-M, Wang J-YW. Development of a real-time clinical decision support system upon the Web MVC-based architecture for prostate cancer treatment. BMC Med Inform Decis Mak 2011;11(01):16

24 Tamposis I, Pouliakis A, Fezoulidis I, Karakitsos P. Mobile platforms supporting health professionals: need, technical requirements, and applications. In: Medical Imaging: Concepts, Methodologies, Tools, and Applications. Hershey, PA: IGI Global; 2017:1020-1043 
25 Pouliakis A, Spathis A, Kottaridi C, et al. Cloud computing for BioLabs. Cloud Computing Applications for Quality Health Care Delivery. Hershey, PA: IGI Global; 2014:228-249

26 Iroju O, Soriyan A, Gambo I, Olaleke J. Interoperability in healthcare: benefits, challenges and resolutions. International Journal of Innovation and Applied Studies. 2013;3(01):262-270

27 Blazona B, Koncar M. HL7 and DICOM based integration of radiology departments with healthcare enterprise information systems. Int J Med Inform 2007;76(Suppl 3):S425-S432

28 Cardoso L, Marins F, Quintas C, et al. Interoperability in healthcare. Health Care Delivery and Clinical Science: Concepts, Methodologies, Tools, and Applications. Hershey, PA: IGI Global; 2018:689-714

29 Goossen W, Langford LH. Exchanging care records using HL7 V3 care provision messages. J Am Med Inform Assoc 2014;21(e2) e363-e368

30 Bidgood WD Jr, Horii SC, Prior FW, Van Syckle DE. Understanding and using DICOM, the data interchange standard for biomedical imaging. J Am Med Inform Assoc 1997;4(03):199-212

31 Flanders AE, Carrino JA. Understanding DICOM and IHE. Semin Roentgenol 2003;38(03):270-281

32 Walters B. VMware virtual platform. Linux journal 1999;1999 (63es):6

33 Warnock MJ, Toland C, Evans D, Wallace B, Nagy P. Benefits of using the DCM4CHE DICOM archive. J Digit Imaging 2007;20(1, suppl 1):125-129

34 Rosset A, Spadola L, Ratib O. OsiriX: an open-source software for navigating in multidimensional DICOM images. J Digit Imaging 2004;17(03):205-216
35 Bender D, Sartipi K. HL7 FHIR: An Agile and RESTful approach to healthcare information exchange. IEEE2013:326-331

36 Mildenberger P, Eichelberg M, Martin E. Introduction to the DICOM standard. Eur Radiol 2002;12(04):920-927

37 Tamposis I, Iordanidis E, Tzortzis L, Papachatzis S. OraHealthX ImaginX A next generation cost effective medical imaging workflow management platform. IEEE2014:406-410

38 Becker M, Böckmann B, Jöckel K-H, et al. Mapping patient data to colorectal cancer clinical algorithms for personalized guidelinebased treatment. Appl Clin Inform 2020;11(02):200-209

39 Melzer G, Maiwald T, Prokosch H-U, Ganslandt T. Leveraging realworld data for the selection of relevant eligibility criteria for the implementation of electronic recruitment support in clinical trials. Appl Clin Inform 2021;12(01):17-26

40 Unberath P, Prokosch HU, Gründner J, Erpenbeck M, Maier C, Christoph J. EHR-independent predictive decision support architecture based on OMOP. Appl Clin Inform 2020;11(03): 399-404

41 Kostrinsky-Thomas AL, Hisama FM, Payne TH. Searching the PDF Haystack: automated knowledge discovery in scanned EHR documents. Appl Clin Inform 2021;12(02):245-250

42 Wang H, Yatawara M, Huang S-C, et al. The integrated proactive surveillance system for prostate cancer. Open Med Inform J 2012; $6: 1-8$

43 Sherman S, Shats O, Ketcham MA, et al. PCCR: pancreatic cancer collaborative registry. Cancer Inform 2011;10:83-91

44 Sherman S, Shats O, Fleissner E, et al. Multicenter breast cancer collaborative registry. Cancer Inform 2011;10:217-226 